\title{
p16, MGMT, RARß2, CLDN3, CRBP and MT1G gene methylation in esophageal squamous cell carcinoma and its precursor lesions
}

\author{
MARK J. ROTH ${ }^{1}$, CHRISTIAN C. ABNET ${ }^{1}$, NAN HU $^{2}$, QUAN-HONG WANG ${ }^{4}$, \\ WENG-QIANG WEI ${ }^{5}$, LISA GREEN ${ }^{3}$, MARY D'ALELIO ${ }^{3}$, YOU-LIN QIAO ${ }^{5}$, \\ SANFORD M. DAWSEY ${ }^{1}$, PHILIP R. TAYLOR ${ }^{2}$ and KAREN WOODSON ${ }^{3}$

\begin{abstract}
${ }^{1}$ Nutritional Epidemiology Branch, ${ }^{2}$ Genetics Epidemiology Branch, Division of Cancer Epidemiology and Genetics; ${ }^{3}$ Cancer Genetics Branch, Center for Cancer Research, National Cancer Institute, Bethesda, MD 20892, USA; ${ }^{4}$ Shanxi Cancer Hospital and Institute, Taiyuan, Shanxi; ${ }^{5}$ Department of Cancer Epidemiology, Cancer Institute, Chinese Academy of Medical Sciences, Beijing, China
\end{abstract}

Received December 5, 2005; Accepted January 18, 2006

\begin{abstract}
Esophageal squamous cell carcinoma (ESCC) is a common cancer with a very poor prognosis. New methods are needed to screen high-risk populations and identify curable tumors and precursor lesions early. Molecular markers may be useful in such screening efforts. This study was designed to determine the prevalence of $p 16, M G M T, R A R \beta 2, C L D N 3$, $C R B P$ and $M T 1 G$ gene methylation in patients with ESCC to evaluate the variation of gene methylation across a spectrum of preneoplastic lesions, and assess the feasibility of using gene methylation in a primary screening test utilizing frozen esophageal cells collected by balloon cytology samplers. Samples were obtained from high-risk subjects from north central China. These samples included 11 foci of histologically normal mucosa, 8 foci of low-grade squamous dysplasia, 7 foci of high-grade squamous dysplasia, and 13 foci of ESCC from 6 fully embedded resection specimens; endoscopic biopsies from 6 individuals with no histological evidence of disease; and frozen esophageal balloon samples from 12 asymptomatic subjects. Promoter $\mathrm{CpG}$ site-specific hypermethylation status was determined for each gene using realtime methylation-specific PCR (qMS-PCR) based on Taqman chemistry. Of the 6 ESCC patients, 5 showed methylation of at least one gene. For most genes, methylation occurred with increasing frequency during neoplastic progression, with the largest increase found between low- and high-grade dysplasia. There was considerable variation in methylation patterns
\end{abstract}

Correspondence to: Dr Mark J. Roth, National Cancer Institute, 6120 Executive Blvd, EPS/320, MSC 7232, Rockville, MD 20892, USA

E-mail:mr166i@nih.gov

Key words: esophagus, squamous cell carcinoma, dysplasia, methylation, p16, MGMT, RARß2, CLDN3, CRBP, MTIG among different foci of the same histological grade, even within individual patients, but 16/20 (80\%) of high-grade dysplastic and cancer foci had $\geq 2$ methylated genes, while $17 / 19$ (89\%) of normal and low-grade dysplastic foci had $<2$ methylated genes. These genes were rarely methylated in histologically normal mucosa from patients with or without ESCC. Gene methylation was common and easily detectable in the frozen esophageal cells collected by balloon cytology samplers. Our data suggest that methylation of $p 16, M G M T$, $R A R \beta 2, C L D N 3, C R B P$, and $M T 1 G$ is common in the esophageal mucosa of patients with ESCC in this high-risk population, and tends to increase in prevalence in foci with increasing histological severity of disease. Methylation data from panels of genes may be able to identify patients with high-grade lesions. Balloon cytology may be able to screen the length of the esophagus effectively for a subset of cells with abnormal methylation, and may be useful in a primary screening test for ESCC and its precursor lesions.

\section{Introduction}

Esophageal squamous cell carcinoma (ESCC) continues to be a significant cause of morbidity and mortality worldwide, and is the fourth most common cause of cancer death in China (1). Within China, the Taihang mountain region in Hebei, Henan and Shanxi Provinces has the highest incidence and mortality rates, reaching more than 100/100,000/year (2). Efforts are ongoing to identify etiologically associated changes in such high-risk areas, so that more effective cancer prevention, screening, and treatment strategies can be developed.

Among the molecular changes potentially associated with esophageal carcinogenesis, there has been considerable interest in gene methylation, the addition of methyl groups to cytosines in $\mathrm{CpG}$ islands, which can silence gene transcription. $\mathrm{CpG}$ islands are 0.5-2.0 kilobase regions rich in cytosine-guanine dinucleotides that are present in the 5 ' region of approximately half of all human genes (3). Methylation of 
Table I. PCR primers/hybridization probes used for qMS-PCR gene-specific DNA hypermethylation assays.

\begin{tabular}{|c|c|c|}
\hline Gene & Location $^{\mathrm{a}}$ & Primer and probe sequences ${ }^{b}$ \\
\hline \multirow[t]{3}{*}{ p16 } & -20 to +87 & Sense: 5'-TAG CGG GCG GCG GGG GA-3' \\
\hline & & Anti-sense: 5'-CGC ACC TCC TCT ACC CGA-3' \\
\hline & & Probe: 6FAM-ATG GAG TCG GCG GCG G-MGBNFQ \\
\hline
\end{tabular}

MGMT +20 to +93

Sense: 5-TGC GTT TCG ACG TTT GTA GGT-3'

Anti-sense: 5'-ACC CAA ACA CTC ACC AAA TCG-3'

Probe: 6FAM-TTT GCG GTG CGT ATC G-MGBNFQ

$R A R \beta 2 \quad-193$ to -177

Sense: 5'-AAG GTG AGA AAT AGG AAA GAA AAC GT-3'

Anti-sense: 5'-TCC CAA CAA CCC TAC AAA AAC A-3'

Probe: 6FAM-TTT GTG CGT TCG TTG TT-MGBNFQ

CLDN3 $\quad-240$ to -164

Sense: 5'- GGT AAT TTT TTT ATT TCG GCG TTG T-3'

Anti-sense: 5'-TCC CAC CTT CAC CGA ACG T-3'

Probe: 6FAM-TCG CGT AGG GTT AAG TT- MGBNFQ

$\begin{array}{ll}C B R P & -9 \text { to }+60\end{array}$

Sense: 5'-TTG TTT TCG GAG GGC GTT TA-3'

Anti-sense: 5'-CCG AAA CAA CGA CTA CCG ATA CT-3'

Probe: 6FAM-TTC GGG TCG TTT ATT ATT- MGBNFQ

MT1G $\quad-206$ to -118

Sense: 5'-AAG GTT TAG TAG GGT GAG AGA ATT CG-3'

Anti-sense: 5'-CGC ACA CCG CAA ACG A-3'

Probe: 6FAM- TTT TTC GCG AGT CGG TGC-MGBNFQ

$A C T B$

Reference standard

Sense: 5'-GGT GGA GGT AGT TAG GGT TTA TTT GTA-3'

Anti-sense: 5'-CCA CAA AAT CAC ACT TAA CCT CAT TT-3'

Probe: 6FAM-CAC TTT TAT TCA ACT AAT CTC-MGBNFQ

FAM, reporter dye; MGBNFQ, molecular-groove binding non-fluorescence quencher hybridization probes that enable the use of probes

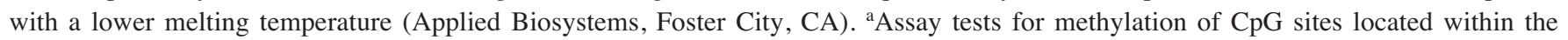
promoter region designated relative to the transcription start site. 'bequences taken from the following GenBank accession numbers: $p 16$, $\mathrm{U} 12818 ; M G M T, \mathrm{X} 61657$; RARß2, 582362; CLDN3, AB000714; CBRP, M11433; MT1G, J-3910; and ACTB, E01094.

these $\mathrm{CpG}$ islands has been reported to cause silencing of several genes in GI cancers, including transcription factors, genes involved in apoptosis, and calcium signaling genes (4). Despite these advances, the role of gene methylation in the neoplastic progression of ESCC remains poorly understood, and its potential use as a primary screening tool for ESCC has not been evaluated.

The understanding of the role of methylation in normal and neoplastic tissues is developing. Quantitative studies show that many highly methylated genes in cancer also have a low but measurable degree of methylation in apparently normal mucosa, and this methylation of apparently normal tissue increases linearly with age (5). Aberrant methylation of multiple genes has been identified in the upper aerodigestive tract epithelium of heavy smokers (6) and in normal tissue adjacent to oral squamous cell carcinomas in those who chew tobacco (7). Aberrant methylation of several genes, including p16, RARß, MGMT and RASSF 1A, has also been identified in ESCC tumors and neighboring normal tissue (8-12).

The current study of ESCC and its preneoplastic lesions focuses on some of the most common and significant genes found to be methylated in other cancers: p16, which alters cell-cycle control (13); O6-MGMT, which impairs DNAdamage repair due to alkylation (13); $R A R \beta$, which modulates growth response (14); $C L D N 3$, which alters cell tight junctions $(15,16)$; $C R B P$, which changes retinol chaperone function (17); and $M T 1 G$, which is involved in metal metabolism and detoxification (18). The aim of this study was to determine the prevalence of methylation of these genes in ESCC patients, evaluate the variation of gene methylation across a spectrum of preneoplastic lesions, and determine the feasibility of using methylation as a component of a primary screening test for the early detection of ESCC and its precursors.

\section{Materials and methods}

Study subjects and samples. All cases and samples were obtained from subjects residing in the Taihang mountain region of north central China. This study was approved by the Institutional Review Boards of the collaborating institutions: Shanxi Cancer Hospital and Institute, Taiyuan, Shanxi Province, China; the Cancer Institute of the Chinese Academy of Medical Sciences (CICAMS), Beijing, China; and the National Cancer Institute, Bethesda, MD, USA. 
Table II. Gene methylation of normal, low-grade dysplasia, high-grade dysplasia and cancer foci from ESCC resection specimens. ${ }^{\mathrm{a}}$

\begin{tabular}{|c|c|c|c|c|c|c|c|c|}
\hline Patient & Histology & p16 & MGMT & RAR $\beta 2$ & CLDN3 & CRBP & MTlG & $\begin{array}{c}\text { \# Genes } \\
\text { methylated }\end{array}$ \\
\hline \multirow{8}{*}{ E7001 } & Normal & & & & & & & 0 \\
\hline & Normal & & & & & & & 0 \\
\hline & Low-grade & & & & & & & 0 \\
\hline & High-grade & & & & & & & 3 \\
\hline & High-grade & & & & & & & 2 \\
\hline & High-grade & & & & & & & 0 \\
\hline & Cancer & & & & & & & 3 \\
\hline & Cancer & & & & & & & 5 \\
\hline \multirow[t]{8}{*}{ E7002 } & Normal & & & & & & & 0 \\
\hline & Normal & & & & & & & 1 \\
\hline & Low-grade & & & & & & & 2 \\
\hline & Low-grade & & & & & & & 2 \\
\hline & High-grade & & & & & & & 3 \\
\hline & Cancer & & & & & & & 3 \\
\hline & Cancer & & & & & & & 6 \\
\hline & Cancer & & & & & & & 3 \\
\hline \multirow{5}{*}{ E7003 } & Normal & & & & & & & 0 \\
\hline & Low-grade & & & & & & & 0 \\
\hline & Low-grade & & & & & & & 0 \\
\hline & Cancer & & & & & & & 0 \\
\hline & Cancer & & & & & & & 0 \\
\hline & & & & & & & & \\
\hline \multirow[t]{5}{*}{ E7012 } & Normal & & & & & & & 0 \\
\hline & Normal & & & & & & & 1 \\
\hline & Low-grade & & & & & & & 0 \\
\hline & Cancer & & & & & & & 3 \\
\hline & Cancer & & & . & & & & 2 \\
\hline & & & & & & & & \\
\hline \multirow[t]{8}{*}{ E7013 } & Normal & & & & & & & 0 \\
\hline & Normal & & & & & & & 0 \\
\hline & Low-grade & & & & & & & 0 \\
\hline & Low-grade & & & & & & & 0 \\
\hline & High-grade & & & & & & & 4 \\
\hline & High-grade & & & & & & & 3 \\
\hline & Cancer & & & & & & & 1 \\
\hline & Cancer & & & & & & & 2 \\
\hline \multirow[t]{5}{*}{ E7017 } & Normal & & & & & & & 0 \\
\hline & Normal & & & & & & & 0 \\
\hline & High-grade & & & & & & & 2 \\
\hline & Cancer & & & & & & & 3 \\
\hline & Cancer & & & & & & & 5 \\
\hline
\end{tabular}

${ }^{a}$ Black and white boxes indicate methylated and unmethylated status, respectively, for each gene. Methylation status was determined by qMSPCR.

Resection specimens from 6 ESCC patients, 5 males and a female with an average age of 61 years (range, 43-71 years) treated at the Shanxi Cancer Hospital in Taiyuan, Shanxi Province, were fully blocked and stored at $-70^{\circ} \mathrm{C}$ until methylation assays could be performed. Serial 5-micron frozen sections were cut from each tissue block and representative foci of normal mucosa $(\mathrm{N}=11)$, low-grade dysplasia $(\mathrm{N}=8)$, high-grade dysplasia $(\mathrm{N}=7)$, and invasive squamous cell carcinoma $(\mathrm{N}=13)$ were chosen based on the histological review of hematoxylin-and-eosin-stained slides by a pathologist (M.J.R.) using accepted histological criteria (19).
Esophageal biopsies from 4 males and 2 females with an average age of 53 years (range, 51-56 years), and balloon cytology cell samples from 5 males and 7 females with an average age of 53 years (range, 43-60 years) were obtained from asymptomatic participants in a cytology screening study in Linxian County, Henan Province (20). The 5-micron sections were cut from ethanol-fixed, paraffin-embedded biopsies from 6 individuals with no endoscopic or histological evidence of disease, and 125- $\mu 1$ aliquots of frozen cytology cell samples were selected from twelve participants with no endsocopic or histological evaluation. 
Table III. Gene methylation in ESCC resections, by histological diagnosis ${ }^{\mathrm{a}}$.

\begin{tabular}{|c|c|c|c|c|c|c|c|}
\hline Histology & No. of foci & p16 & MGMT & RARß2 & CLDN3 & CRBP & MT1G \\
\hline Normal & 11 & $0(0 \%)$ & $1(9 \%)$ & $0(0 \%)$ & $0(0 \%)$ & $0(0 \%)$ & $1(9 \%)$ \\
\hline Low-grade dysplasia & 8 & $1(12 \%)$ & $1(12 \%)$ & $0(0 \%)$ & $1(12 \%)$ & $0(0 \%)$ & $1(12 \%)$ \\
\hline High-grade dysplasia & 7 & $2(29 \%)$ & $4(57 \%)$ & $3(43 \%)$ & $2(29 \%)$ & $2(29 \%)$ & $4(57 \%)$ \\
\hline Cancer & 13 & $5(39 \%)$ & $6(46 \%)$ & $5(39 \%)$ & $9(69 \%)$ & $4(31 \%)$ & $8(62 \%)$ \\
\hline
\end{tabular}

${ }^{a}$ Multiple histological foci were evaluated from 6 fully embedded esophageal resection specimens. The result of the Chi-square test comparing low risk $(\mathrm{N}+\mathrm{LG})$ versus high risk $(\mathrm{HG}+\mathrm{C})$ histologies was $\mathrm{p} \leq 0.02$ for each gene

\section{ESCC Gene Methylation}

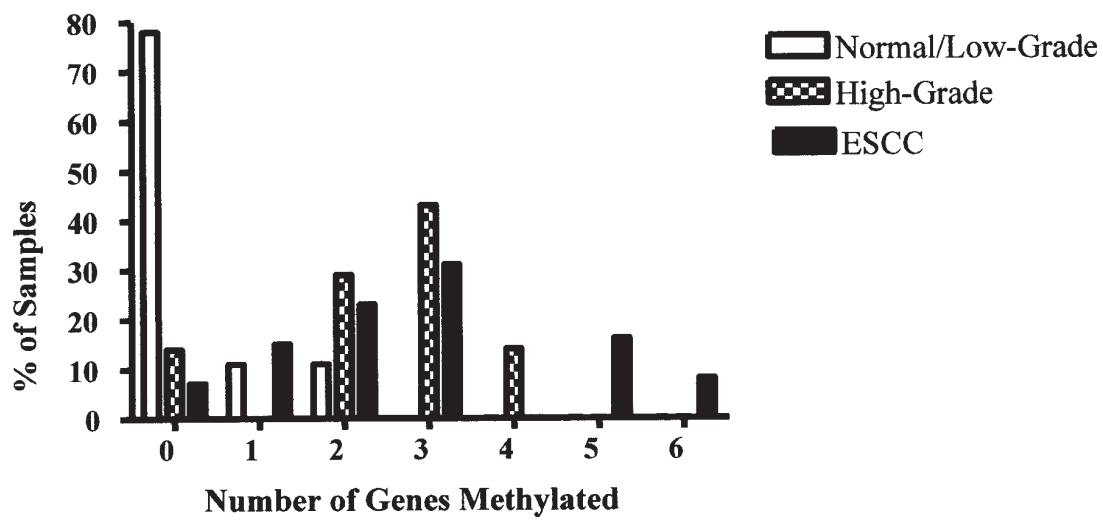

Figure 1. Number of genes methylated in normal/low-grade, high-grade, and cancer foci from six ESCC resection specimens

Bisulfite modification and real-time methylation-specific $P C R$. Genomic DNA was purified from frozen esophagectomy specimens (one 5-micron section/block), ethanol-fixed, paraffin-embedded endoscopic biopsy specimens (ten 10micron sections/biopsy) and frozen cytology specimens (125 $\mu 1$ each) using the DNeasy kit (Qiagen, Valencia, CA). The frozen sections were sequentially rinsed for $10 \mathrm{sec}$ in $70 \%, 95 \%$, and $100 \%$ ethanol before being placed in lysis buffer. The fixed biopsy specimens were deparaffinized in xylene (twice for $5 \mathrm{~min}$ ) and rinsed in $100 \%$ ethanol, followed by the addition of lysis buffer. The cytology samples were centrifuged at 10,000 rpm for $2 \mathrm{~min}$ and resuspended in lysis buffer. All samples were lysed for $48 \mathrm{~h}$ (with a second aliquot of proteinase $\mathrm{K}$ added after $24 \mathrm{~h}$ ) and processed following the manufacturer's instructions.

The gene-specific hypermethylation status was determined using methylation-specific PCR (qMS-PCR) based on Taqman chemistry (Applied Biosystem, Foster City, CA) as previously described $(21,22)$. Bisulfite modification was performed as previously described (22). Primers and hybridization probes were designed to bind specifically to bisulfite-converted sequences of $\mathrm{CpG}$ islands in the promoters of each gene, with each assay evaluating 4-6 individual $\mathrm{CpG}$ sites. The primers and probes for each gene as well as their location in the promoter are listed in Table I.

The qMS-PCR reactions were carried out in a volume of $10 \mu 1$, using Taqman Universal PCR Master Mix and No
AmpErase UNG (Applied Biosystems, Foster City, CA). Each PCR reaction mixture contained $300 \mathrm{nM}$ of each primer, $100 \mathrm{nM}$ probe, and 1X Taqman buffer. Amplification and detection were carried out using the following profile: one step at $50^{\circ} \mathrm{C}$ for $2 \mathrm{~min}$, one step at $95^{\circ} \mathrm{C}$ for $10 \mathrm{~min}$, and 50 cycles at $95^{\circ} \mathrm{C}$ for $15 \mathrm{sec}$ and $60^{\circ} \mathrm{C}$ for $1 \mathrm{~min}$. The sensitivity and specificity of the assays were tested by running standards of serial dilutions with known amounts of methylated DNA (artificially methylated human placental DNA using E. coli SSI methyl-transferase) ranging from 10.0 to $0.01 \mathrm{ng}$ and unmethylated DNA. The amount of hypermethylated DNA was determined by reading the midpoint of the linear portion of the S-shaped real-time curves, called the $\mathrm{Ct}$ point or threshold cycle. The $\mathrm{Ct}$ refers to the number of cycles it takes a sample to reach a specific fluorescence threshold. Samples with a $\mathrm{Ct}$ below 45 were considered to have promoter-region hypermethylation. All samples were well within the assay's range of sensitivity and reproducibility based on an internal reference standard (ACTB) (Ct scores below 40 for input DNA). All samples were run in duplicate, and the average $\mathrm{Ct}$ value was used. Template controls were not run in duplicate for each assay.

Statistical analysis. All statistical analyses were performed on Stata 8.0 (College Station, TX). The Chi-square test was used to test for differences in the frequency of methylation for each gene between the normal/low-grade versus high- 
Table IV. Gene methylation in esophageal balloon cytology specimens from asymptomatic inhabitants of a high-risk ESCC region $(\mathrm{N}=12)^{\mathrm{a}}$.

\begin{tabular}{|c|c|c|c|c|c|c|c|}
\hline Patient & $\overline{\text { p16 }}$ & MGMT & $\operatorname{RAR} \beta 2$ & CLDN3 & CRBP & MT1G & $\begin{array}{c}\text { \# Genes } \\
\text { methylated }\end{array}$ \\
\hline $7-747$ & & & & & & & 0 \\
\hline $7-772$ & & & & & & & 2 \\
\hline $7-892$ & & & & & & & 5 \\
\hline $7-766$ & & & & & & & 2 \\
\hline $7-856$ & & & & & & & 0 \\
\hline $7-801$ & & & & & & & 6 \\
\hline $7-069$ & & & & & & & 4 \\
\hline $7-072$ & & & & & & & 3 \\
\hline $7-113$ & & & & & & & 1 \\
\hline $7-141$ & & & & & & & 1 \\
\hline $7-297$ & & & & & & & 3 \\
\hline $7-313$ & & & & & & & 3 \\
\hline $\begin{array}{l}\text { Overall } \\
\text { Prevalence }\end{array}$ & $2(17 \%)$ & $4(33 \%)$ & $2(17 \%)$ & $9(75 \%)$ & $6(50 \%)$ & $7(58 \%)$ & \\
\hline
\end{tabular}

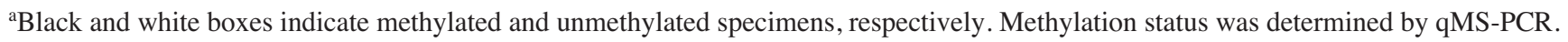

grade/cancer groups. The Wilcoxon rank sum test was used to test for differences in the number of genes methylated across normal/low-grade versus high-grade/cancer groups.

\section{Results}

The methylation status of six genes, p16, MGMT, RARß2, $C L D N 3, C R B P$, and $M T 1 G$, was analyzed in histological foci comprising a range of disease including normal, low-grade squamous dysplasia, high-grade squamous dysplasia, and invasive ESCC from six esophageal cancer resections. Table II shows the gene methylation status of these foci by histological diagnosis and patient, including one case (E7003) in which no methylation was identified. Thus, 5 of the 6 patients $(83 \%)$ showed methylation of at least one of the six genes tested. Three patients $(50 \%)$ had gene methylation involving only high-grade dysplasia and tumor foci, while 2 patients (33\%) showed gene methylation in normal and/or low-grade dysplasia foci as well. Only two foci of histologically normal mucosa showed gene methylation, one from $M G M T$ and one from $M T 1 G$. Gene methylation was commonly heterogeneous across multiple foci with the same histological diagnosis from the same resection specimen. In fact, the only time that such foci had identical methylation profiles for all six genes was when they showed no methylation (Table II).

Summary data for gene methylation by histological diagnosis is presented in Table III. With minor exceptions, gene methylation occurred with increasing frequency during neoplastic progression from normal to low-grade dysplasia to high-grade dysplasia to invasive cancer. For five of the six genes, the largest increase in methylation frequency occurred between low-grade and high-grade dysplasia, and a Chi-square test comparing methylation frequency between normal/lowgrade and high-grade/cancer foci was significant for each gene $(\mathrm{p} \leq 0.02)$.

The distribution of the number of methylated genes by histological diagnosis is presented in Fig. 1, which shows differences between normal/low-grade dysplastic lesions, high-grade dysplastic lesions, and cancer foci. The median number of methylated genes was 0.2 for normal, 0.5 for lowgrade dysplasia, 2.4 for high-grade dysplasia, and 2.8 for cancer. Some 16/20 (80\%) of high-grade dysplastic and cancer foci had $\geq 2$ methylated genes, while 17/19 (89\%) of normal and low-grade dysplastic foci had $<2$ methylated genes.

Gene methylation was also assessed in endoscopic biopsies from 6 individuals with no endoscopic or histological evidence of disease. The findings were similar to those in the histologically normal foci from the esophagectomy specimens: there was no methylation of $p 16, R A R \beta 2, C L D N 3$, or $C R B P$, and only a single individual showed methylation of $M G M T$ and $M T 1 G$.

The methylation status of 12 esophageal balloon cytology specimens collected from asymptomatic high-risk individuals with no endoscopic or histological evaluation was analyzed as part of an initial test of the feasibility of using gene methylation in such samples in a primary cancer screening test 
(Table IV). Gene methylation was identified in all but two specimens, with methylation of $p 16$ and $R A R \beta 2$ occurring in $17 \%$ of the samples, MGMT in $33 \%$, and CLDN3, MT1G and $C R B P$ in at least $50 \%$. Esophageal balloon cytology samples from $4(33 \%)$ patients had zero or one methylated gene, $5(42 \%)$ had two or three methylated genes, and 3 $(25 \%)$ had four, five or six methylated genes.

\section{Discussion}

This study examined the prevalence of methylation of the p16, MGMT, RARß2, CLDN3, CRBP, and MT1G genes in tumor tissue and associated neoplastic precursor lesions from patients with esophageal squamous cell carcinoma (ESCC). To accomplish this, a spectrum of histologies from fully embedded ESCC resections was examined. This study also explored the possibility of a methylation 'field effect' by comparing: i) methylation patterns in foci of different histological grades from each individual, ii) separate foci of a single histological grade from a single individual, and iii) histologically normal foci from cancer resections and normal endoscopic biopsies from individuals with no histological evidence of disease. The practicality of using gene methylation as part of a primary screening tool was also evaluated by examining esophageal balloon cytology cell samples from asymptomatic individuals living in a high-risk region.

All but one of the esophageal resection specimens showed methylation in at least one of the genes evaluated in this study, suggesting that methylation of these genes is a common occurrence in the neoplastic development of ESCC in this population. There was a tendency for the methylation of each tested gene to increase with histological progression, with the clearest and most consistent increase occurring between lowgrade and high-grade dysplasia. There was a significantly higher frequency of methylation in high-grade dysplasia and cancer foci compared to low-grade dysplasia and normal foci ( $p \leq 0.02$ for each gene), and essentially no difference in methylation frequency between high-grade dysplasia and cancer in five of the six genes.

Evidence of a methylation 'field effect' was mixed. While some genes (MGMT, MT1G) were occasionally methylated across a spectrum of three or more histological grades in single resection specimens, the other genes were not, and the heterogeneity of methylation patterns in different foci of the same histological grade in individual resection specimens was striking. In addition, the pattern of gene methylation in histologically normal foci from patients with and without cancer was quite similar; there was no methylation of $p 16$, $R A R \beta 2, C L D N 3$ or $C R B P$, and only rare methylation of $M G M T$ or $M T 1 G$. While this is consistent with the latter two genes occasionally being methylated early in the neoplastic process, it is also consistent with these genes occasionally being methylated in normal esophageal mucosa during aging, and does not suggest a widespread 'field' of aberrant methylation preferentially spreading into histologically normal mucosa in cancer patients. Prospective studies will be needed to determine the significance of gene methylation in the absence of histologically identifiable disease.

Only three previous studies have evaluated the methylation of any of these genes across a histological progression of normal, squamous dysplasia and ESCC tumor tissues. Tokugawa et al examined p16 methylation in 42 ESCC resection specimens and evaluated foci of invasive tumors, high-grade dysplasia, adjacent normal tissue and remote normal tissue in each case (9). They found p16 methylation in $30(71 \%), 30(71 \%), 24(57 \%)$, and $0(0 \%)$ of these foci, respectively. Strikingly, they found the same methylation status in all blocks of tumor and dysplasia in each individual case, and all methylation-positive normal tissue was adjacent to methylation-positive tumors, findings that are consistent with totally homogeneous 'fields' of aberrant p16 methylation. In contrast, we found p16 methylation in $50 \%, 17 \%$ and $0 \%$ of ESCC, high-grade dysplasia and normal tissue and considerable heterogeneity in methylation status among the tumor and dysplasia blocks from individual cases.

Wang et al examined RAR $\beta 2$ methylation in tumor and neighboring non-tumor samples from ESCC patients (10). They found methylation in 14/20 (70\%) ESCC samples, 7/12 (58\%) dysplasia samples, and 2/17 (12\%) normal foci. Only 5 cases evaluated all three histologies in the same patient. In these cases, all of the normal tissues were unmethylated, and the methylation status of the dysplastic and tumor tissue was concordant in only 3 of the 5 patients. Similarly, we found no $R A R \beta 2$ methylation in normal blocks and inconsistent methylation in dysplasia and tumor tissue.

The current study differs from that of Nie et al (8), partly because the majority of samples in the latter are basal cell hyperplasia $(\mathrm{BCH}) . \mathrm{BCH}$ has a relatively low risk of progressing into cancer (23) and, consequently, the biological significance of gene methylation associated with this histological category may be less clear. The current study analyzes endoscopic biopsies representing an individual's worst histological diagnosis after mucosal iodine screening and multiple biopsies, whereas the earlier study does not specify whether multiple biopsies were taken from each individual or if analysis was made of their worst histological diagnosis. In addition, given the potential for etiologically significant interplay between the epithelium and underlying stroma (24), our specimens were not microdissected, in contrast to Nie et al. Lastly, according to the findings of Nie et al, once a gene is methylated, it can be found methylated in other biopsies from the same patient containing a more severe histology. This differs from the heterogeneity found in our esophageal resections.

We have previously shown that squamous dysplasia is the clinically important precursor lesion of ESCC in this highrisk Chinese population (23), squamous dysplasia (especially high-grade dysplasia) is usually endoscopically visible after mucosal iodine staining (25), which allows it to be targeted for biopsy and treatment, and high-grade dysplastic and early invasive lesions are usually treatable by focal endoscopic therapy (26). An accurate, cost-effective primary screening test that can identify patients with dysplastic or worse lesions who need endoscopy is still required for a clinically practical early detection and treatment program in such high-risk populations. Earlier studies of esophageal balloon cytology (EBC) have shown that morphological diagnosis of the collected cells is not sufficiently accurate for this purpose (maximum sensitivity/specificity of $58 \% / 65 \%$ for biopsyproven squamous dysplasia or cancer) $(20,27)$. The current 
study, while small, shows a potentially useful way to discriminate high-grade dysplastic or worse foci from foci with less than high-grade dysplasia by evaluating methylation in a panel of six genes: $\geq 2$ methylated genes had a sensitivity/specificity of $80 \% / 89 \%$ for identifying foci with high-grade dysplasia or worse disease. We also showed the feasibility of measuring methylation in frozen cells collected by EBC. Together, these findings raise the possibility that the measurement of methylation patterns may be a useful component of a practical primary screening test for squamous dysplasia and ESCC.

In summary, methylation of $p 16, M G M T, R A R \beta 2, C L D N 3$, $C R B P$ and $M T 1 G$ genes is common in the esophageal mucosa of patients with ESCC, and tends to increase in prevalence in mucosal foci as the histological severity of the disease increases. These genes are typically unmethylated in histologically normal mucosa from patients with or without ESCC. There is considerable variation in methylation patterns among different mucosal foci of the same histological grade, even in individual patients, but methylation patterns in panels of genes may be able to identify individual patients with high-grade lesions. Methylation can be detected in frozen cells collected by EBC samplers, suggesting that it may be useful in a primary screening test for ESCC and its precursor lesions.

\section{Acknowledgements}

This research was supported by the Intramural Research Program of the NIH, National Cancer Institute and an NIH research contract (\#N01-SC-91019).

\section{References}

1. Yang L, Parkin DM, Li LD, Chen YD and Bray F: Estimation and projection of the national profile of cancer mortality in China: 1991-2005. Br J Cancer 90: 2157-2166, 2004.

2. Ke L: Mortality and incidence trends from esophageal cancer in selected geographic areas of China circa 1970-90. Int J Cancer 102: 271-274, 2002.

3. Rashid A and Issa JPJ: CpG island methylation in gastroenterologic neoplasia: a maturing field. Gastroenterology 127 : 1578-1588, 2004.

4. Laird PW: The power and the promise of DNA methylation markers. Nat Rev 3: 253-266, 2003.

5. Issa JP: Epigenetic variation and human disease. J Nutr 132: 2388S-2392S, 2002.

6. Zochbauer-Muller S, Lam S, Toyooka S, Virmani AK, Toyooka K, Seidl S, Minna JD and Gazdar AF: Aberrant methylation of multiple genes in the upper aerodigestive tract epithelium of heavy smokers. Int J Cancer 107: 612-616, 2003.

7. Kulkarni V and Saranth D: Concurrent hypermethylation of multiple regulatory genes in chewing tobacco associated oral squamous cell carcinomas and adjacent normal tissues. Oral Oncology 40: 145-153, 2004.

8. Nie Y, Liao J, Zhao X, Song Y, Yang G-Y, Wang L-D and Yang CS: Detection of multiple gene hypermethylation in the development of esophageal squamous cell carcinoma. Carcinogenesis 23: 1713-1720, 2002.

9. Tokugawa T, Sugihara H, Tani T and Hattori T: Modes of silencing of p16 in development of esophageal squamous cell carcinoma. Cancer Res 62: 4938-4944, 2002.
10. Wang Y, Fang MZ, Liao J, Yang G-Y, Nie Y, Song Y, So C, $\mathrm{Xu}$ X, Wang L-D and Yang CS: Hypermethylation-associated inactivation of retinoic acid receptor $\beta$ in human esophageal squamous cell carcinoma. Clin Cancer Res 9: 5257-5263, 2003.

11. Zhang L, Lu W, Miao X, Xing D, Tan W and Lin D Inactivation of DNA repair gene $\mathrm{O}^{6}$-methylguanine-DNA methyltransferase by promoter hypermethylation and its relation to p53 mutations in esophageal squamous cell carcinoma. Carcinogenesis 24: 1039-1044, 2003.

12. Kuroki T, Trepasso F, Yendamuri S, Matsuyama A, Alder H, Mori M and Croce CM: Promotor hypermethylation of RASSF1A in esophageal squamous cell carcinoma. Clin Cancer Res 9: 1441-1445, 2003.

13. Esteller M: Aberrant DNA methylation as a cancer-inducing mechanism. Ann Rev Pharmacol Toxicol 45: 629-656, 2005

14. Herman JG and Baylin SB: Gene silencing in cancer in association with promoter hypermethylation. NEJM 349: 2042-2054, 2003.

15. Coyne CB, Gambling TM, Boucher RC, Carson JL and Johnson LG: Role of claudin interaction in airway tight junctional permeability. AJP-Lung 285: 1166-1178, 2003.

16. Langbein L, Pape UF, Grund C, Praetzel S, Moll I, Moll R and Franke WW: Tight junction-related structures in the absence of a lumen: occluding, claudins and tight junction plaque proteins in densely packed cell formations of stratified epithelia and squamous cell carcinomas. Eur J Cell Biol 82: 385-400, 2003.

17. Estellar M, Guo M, Moreno V, Peinado MA, Capella G, Galm O, Baylin SB and Herman JG: Hypermethylationassociated inactivation of the cellular retinol-binding protein I gene in human cancer. Cancer Res 62: 5902-5905, 2002.

18. Andrews GK: Regulation of metallothionein gene expression by oxidative stess and metal ions. Biochem Pharmacol 59: 95-104, 2000.

19. Dawsey SM, Lewin KJ, Liu FS, Wang GQ and Shen Q Esophageal morphology from Linxian, China. Squamous histologic findings in 754 patients. Cancer 73: 2027-2037, 1994.

20. Guo G, Roth MJ, Pan Q-J, Wang G-Q, Kochman ML, Qiao Y-L, Henry MR, Wei W-Q, Giffen CA, Lu N, Abnet CC, Hao C-Q, Taylor PR and Dawsey SM: Cytologic detection of esophageal squamous cell carcinoma and precursor lesions using balloon samplers and liquid-based cytology in asymptomatic adults in Linxian, China. Gastroenterology 128: A1004, 2005.

21. Woodson K, Hayes R, Wideroff L, Viallruz L and Tangrea J: Hypermethylation of GSTP1, CD44, and E-cadherin genes in prostate cancer among U.S. blacks and whites. Prostate 55: 199-205, 2003.

22. Woodson K, Gillespie J, Hanson J, Emmert-Buck M, Phillips JM, Linehan WM and Tangrea JA: Heterogeneous gene methylation patterns among pre-invasive and cancerous lesions of the prostate: a histopathologic study of whole mount prostate specimens. Prostate 60: 25-31, 2004.

23. Wang GQ, Abnet CC, Shen Q, Lewin KJ, Sun XD, Roth MJ, Qiao YL, Mark SD, Dong ZW, Taylor PR and Dawsey SM: Histologic precursors of esophageal squamous cell carcinoma: results from a 13-year prospective follow-up study in a high-risk population. Gut 54: 187-192, 2005.

24. Kim JB, Stein R and O'Hare MJ: Tumour-stromal interactions in breast cancer: the role of stroma in tumorigenesis. Tumor Biol 26: 173-185, 2005

25. Dawsey SM, Fleischer DE, Wang GQ, Zhou B, Kidwell JA, Lu N, Lewin KJ, Roth MJ, Tio TL and Taylor PR: Mucosal iodine staining improves endoscopic visualization of squamous dysplasia and squamous cell carcinoma of the esophagus in Linxian, China. Cancer 83: 220-231, 1998.

26. Fleischer DE, Wang GQ, Dawsey SM, Wang GQ, Strader DB, Liu PY, Zhou B, Barros SGS and Krebs R: Endoscopic therapy for esophageal dysplasia (ED) and early esophageal cancer (EEC) in Linxian, China, 1998: Implications for America. Gastrointest Endosc 49: AB102, 1999.

27. Roth MJ, Liu SF, Dawsey SM, Zhou B, Copeland C, Wang GQ, Solomon D, Baker SG, Giffen CA and Taylor PR: Cytologic detection of esophageal squamous cell carcinoma and precursor lesions using balloon and sponge samplers in asymptomatic adults in Linxian, China. Cancer 80: 2047-2059, 1997. 